\title{
Simultaneous Prediction of Wrist/Hand Motion via Wearable Ultrasound Sensing
}

\author{
Xingchen Yang, Student Member, IEEE, Jipeng Yan, Student Member, IEEE, Yinfeng Fang, Member, IEEE, \\ Dalin Zhou, Member, IEEE, and Honghai Liu*, Senior Member, IEEE
}

\begin{abstract}
The ability to predict wrist and hand motions simultaneously is essential for natural controls of hand protheses. In this paper, we propose a novel method that includes subclass discriminant analysis (SDA) and principal component analysis for the simultaneous prediction of wrist rotation (pronation/supination) and finger gestures using wearable ultrasound. We tested the method on eight finger gestures with concurrent wrist rotations. Results showed that SDA was able to achieve accurate classification of both finger gestures and wrist rotations under dynamic wrist rotations. When grouping the wrist rotations into three subclasses, about $99.2 \pm 1.2 \%$ of finger gestures and $92.8 \pm 1.4 \%$ of wrist rotations can be accurately classified. Moreover, we found that the first principal component (PC1) of the selected ultrasound features was linear to the wrist rotation angle regardless of finger gestures. We further used PC1 in an online tracking task for continuous wrist control and demonstrated that a wrist tracking precision $\left(R^{2}\right)$ of $0.954 \pm$ 0.012 and a finger gesture classification accuracy of $96.5 \pm 1.7 \%$ can be simultaneously achieved, with only two minutes of user training. Our proposed simultaneous wrist/hand control scheme is training-efficient and robust, paving the way for musculaturedriven artificial hand control and rehabilitation treatment.
\end{abstract}

Index Terms-Subclass discriminant analysis, principal component analysis, simultaneous wrist/hand control, wearable ultrasound sensing.

\section{INTRODUCTION}

Artificial hand prostheses have evolved from one degree of freedom (DoF) gripper to multi-finger hands with multiple DoFs. To enable intuitive control of dexterous prostheses, wearable hand gesture recognition has been widely studied to identify the relationship between muscle activities and intended hand gestures. Currently, the prevailing way for wearable hand gesture recognition is myoelectric pattern recognition, by which multi-DoF hand motions can be decoded from surface electromyography (sEMG) signals sequentially. However, this strategy can only predict one gesture at a time, rather than simultaneous prediction of multiple DoFs such as combined wrist/hand motions. This results in the gesture control being counterintuitive, since natural limb movement usually consists of simultaneous activations of multiple DoFs.

This work was supported by the National Natural Science Foundation of China (Grant Nos. 51575338, 61733011).

Yang, Yan, Liu are with the State Key Laboratory of Mechanical System and Vibration, School of Mechanical Engineering, Shanghai Jiao Tong University, Shanghai, China. (e-mail: xingchen.yang@sjtu.edu.cn).

Fang is with School of Communication Engineering, Hanzhou Dianzi University, Hangzhou, China.

Zhou, Liu are with School of Computing, University of Portsmouth, PO1 3HE, Portsmouth, UK. (e-mail: honghai.liu@ port.ac.uk).
Several approaches have been explored to associate muscle activation patterns to multi-DoF hand motions. Regression techniques are preferred in this field, through which simultaneous and proportional multi-DoF control (SPC) can be achieved [1], [2]. In addition, muscle synergy inspired nonnegative matrix factorization provides another way for the SPC [3]. However, most of these studies focused on the simultaneous prediction of multi-DoF wrist motions or finger motions, instead of combined wrist/hand motions frequently used in daily life.

In the context of simultaneous wrist/hand control, some sEMG-based classification methods have been explored. Davidge et al. used a single linear discrimination analysis (LDA) model to classify combined wrist/hand motions, where both individual (1-DoF) motions and combined motions (multi-DoF) were labeled as different classes [4]. While remarkable classification accuracy can be achieved in this way, extensive training was required since both individual and combined motions were needed for the model calibration [5]. By contrast, Baker and Boschmann et al. proposed a parallel classification scheme, where multiple classifiers were used in parallel to classify different DoFs simultaneously [6], [7]. The parallel classifiers can be trained with 1-DoF exemplars, but the classification performance was undesirable unless multi-DoF exemplars were included [8]. The parallel classification scheme was later modified by Young et al. to improve its reliability, but the cumbersome training problem remains unsolved [9]. Recently, Nowak et al. proposed a linear enhanced training (LET) strategy to reduce training burden, with the hypothesis that features of combined motions can stem from individual motions [10]. However, initial training of both individual and combined motions with a few subjects was still required to build their relationships. Inspired by this idea, Antuvan et al. attempted an LDA-based algorithm to build the relationship between combined wrist/hand motions and individual motions in two-dimensional space, achieving accurate recognition of both individual and combined motions by training only with individual motions [11]. However, the number of activated DoFs was limited to two in this study. In addition to classification-based methods, some regression techniques have been attempted for the simultaneous prediction of wrist/hand motions. However, these methods usually require a large amount of training data, and their clinical robustness remains unverified [12], [13]. To date, achieving simultaneous wrist/hand control using physiologically related muscle information remains a formidable problem.

While sEMG is still dominant in the field of self-powered 
prosthesis control, its inherent noisy and non-stationary properties hinder its practical applications [14]. Recently, ultrasound has been regarded as an alternative to sEMG for the prosthesis control, due to its ability to precisely monitor muscle deformations. Since functional muscles related to finger motions and wrist motions are seated in different depths and discernable by ultrasound signals, it is possible to discriminate activations of wrist and hand motions simultaneously using ultrasound. Early studies have demonstrated that both discrete finger [15]/wrist motions [16] and continuous finger flexion [14]/wrist extension [17] can be decoded from Bmode ultrasound images, with better performance than sEMG [14]. Moreover, it is feasible to predict fine finger motions via one-dimensional A-mode ultrasound [18], [19], which is more lightweight and wearable compared to the bulky B-mode ultrasound. However, so far no study has concentrated on the prediction of simultaneous wrist/hand motion via ultrasound.

In this paper, we report a wearable A-mode ultrasound based method, to realize simultaneous wrist/hand motion prediction. Specifically, we propose a novel method that includes subclass discriminant analysis (SDA) and principal component analysis (PCA) for the simultaneous prediction of wrist rotation (pronation/supination) and finger gestures. With the established models, eight dexterous finger gestures can be accurately predicted during wrist rotations, and corresponding wrist rotation positions can be precisely estimated at the same time in either discrete or continuous manners (see Fig. 1). Both offline analysis and online validation were conducted in this study, to assess the feasibility of ultrasound-based simultaneous wrist/hand motion prediction.

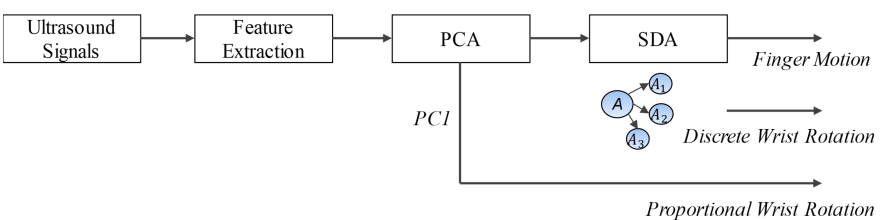

Fig. 1. Illustration of the simultaneous wrist/hand motion prediction scheme. SDA denotes subclass discriminant analysis, PCA denotes principal component analysis, and PC1 denotes the first principal component.

\section{Methods}

\section{A. Subjects}

Eight healthy subjects (all male; $24.6 \pm 1.6$ years; $64.3 \pm$ $5.6 \mathrm{~kg} ; 174.1 \pm 4.3 \mathrm{~cm}$ ) volunteered for this study. None of them had a history of neuromuscular or joint diseases. All subjects provided informed consent prior to participating in the experiment. The testing procedure was in accordance with the Declaration of Helsinki.

\section{B. Offline Experiment}

The subjects sat naturally, relaxed their elbows on the table and kept their palms upwards. The angle between the upper arm and the forearm was about $120^{\circ}$. Eight A-mode ultrasound transducers $(\varnothing 9 \times 11 \mathrm{~mm})$ were placed around the forearm with a customized armband [20], approximately $10 \mathrm{~cm}$ distal to the elbow. The positions of transducers are shown in Fig. 2, where the distance between channel 1 and channel 8 was a little larger and the others were equidistant.

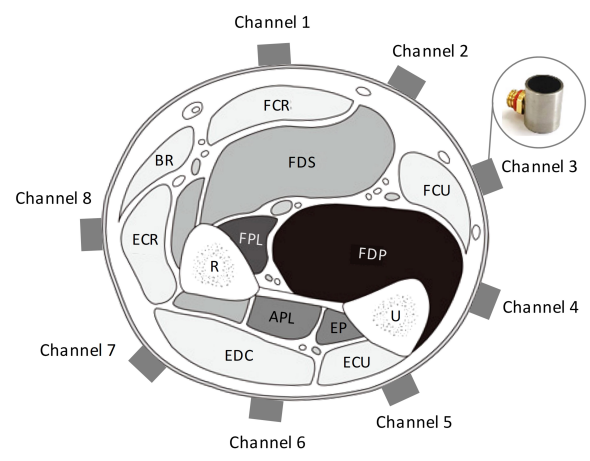

Fig. 2. Transducer placement. $\mathrm{R}=$ radius; $\mathrm{U}=$ ulna; $\mathrm{BR}=$ brachioradialis; $\mathrm{FCU}=$ flexor carpi ulnaris; FCR = flexor carpi radialis; FDP = flexor digitorum profundus; FDS = flexor digitorum superficialis; FPL = flexor pollicis longus; $\mathrm{APL}=$ abductor pollicis longus; $\mathrm{EP}=$ extensor pollicis longus and brevis; $\mathrm{ECU}=$ extensor carpi ulnaris; ECR = extensor carpi radialis; $\mathrm{EDC}=$ extensor digitorum communis.

The transducers were sequentially driven by a customized wearable ultrasound system [20], with a frame rate of $10 \mathrm{~Hz}$, a sampling rate of $20 \mathrm{MHz}$, and sampling dots of 1000 for each channel. In addition, a commercial inertial measurement unit (IMU) sensor (Xsens-MTi-100, Xsens Technologies B.V., Netherlands) was attached on the ventral side of the wrist to record the wrist rotation angle, sampling at $100 \mathrm{~Hz}$. The collection of ultrasound and IMU signals was simultaneously triggered by a software (Quick Macro, Chuangyi Jiahe Soft Co., Ltd., China).

Wrist rotations and eight types of finger gestures were studied in this work, including rest (RS), fist (FS), index point (IP), fine pinch (FP), tripod grip (TG), key grip (KG), peace sign (PS), and hang loose (HL), as shown in Fig. 3. During the offline experiment, subjects were instructed to perform a dynamic training; each finger gesture was performed for 30 seconds with continuous wrist rotations at a rhythm of approximately $0.5 \mathrm{~Hz}$. To avoid fatigue, there was a 10-second rest between two continuous finger gestures. Low level of muscle activation was used throughout, given that ultrasoundbased gesture recognition was shown to be insensitive to muscle activation levels [21].

\section{Online Test}

A custom-built graphical user interface (GUI) to perform a tracking task was designed to qualify the performance of the simultaneous wrist/hand control. In the tracking task, subjects were instructed to perform a target finger gesture and to control a black cursor to trace a target waveform with wrist rotations.

Two kinds of tracking missions were studied. The first was tracking discrete platforms through discrete wrist rotations by SDA (see Fig. 10A). The second was tracking continuous sine waves through continuous wrist rotations by PCA (see Fig. 10B and Fig. 3, $0.1 \mathrm{~Hz}$ sine wave, where the negative values were set to 0 ). Four subjects participated in the first 
tracking mission, and all the subjects participated in the second.

Before the online test, two minutes of dynamic training were required. There were three trials in total. In each trial, each gesture was performed for 5 seconds with continuous wrist rotations at a rhythm of approximately $0.5 \mathrm{~Hz}$. During the online test, each gesture was tested for 30 seconds sequentially, during which subjects were instructed to hold the testing gesture steadily and to complete the tracking task through wrist rotations. There was a 2 -second preview before the tracking started; hence, the real tracking time of each gesture was 28 seconds. For the continuous tracking task, only the last 23 seconds of data were analyzed for each gesture, because subjects required some time to achieve steady-state tracking performance.

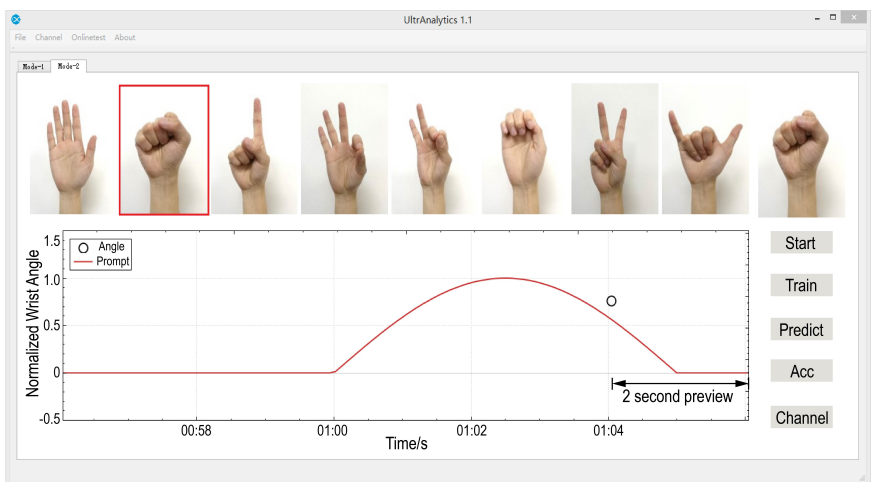

Fig. 3. Custom-built graphic user interface (GUI) for the online wrist/hand motion control. Subjects were instructed to perform a target finger gesture and to control a black curse to trace a target waveform with wrist rotations. The target finger gesture was prompted with a red frame, and the real-time gesture recognition result was displayed on the upper-right corner. From left to right, the finger gestures are rest (RS), fist (FS), index point (IP), fine pinch (FP), tripod grip (TG), key grip (KG), peace sign (PS), and hang loose (HL), respectively. Each finger gesture was tested for 30 seconds sequentially in the online test. There was a 2 -second preview before the tracking started, hence the real tracking time of each gesture was 28 seconds.

\section{Data Processing and Analysis}

1) Feature Extraction: To remove noises and enhance meaningful information, we first preprocessed the raw ultrasound signals with time gain compensation, bandpass filtering, envelope detection, and log compression [21]. Since the first and the last 20 data points of the ultrasound signals carried little meaningful information, we removed them during the preprocessing. The remaining 960 data points of each channel were evenly segmented into 48 windows, with a segmentation length of 20 points. For each window, the following statistical features were analyzed

$$
\begin{aligned}
& f_{1}=\operatorname{mean}(X) \\
& f_{2}=\operatorname{std}(X) \\
& f_{3}=\max (X) \\
& f_{4}=\min (X) \\
& f_{5}=\operatorname{sum}(\operatorname{abs}(\operatorname{diff}(X))) \\
& f_{6}=\operatorname{skewness}(X) \\
& f_{7}=\operatorname{kurtosis}(X)
\end{aligned}
$$

where $X$ is the data of one analysis widow, $f_{1}-f_{7}$ are calculated with Matlab 2016Rb with corresponding functions. For each feature, the values of all the analysis windows and all the channels were connected.

The importance of these features was determined by the Random Forest algorithm, which was created using the TreeBagger function in Matlab 2016Rb. Then, two types of significant features were concatenated together for the subsequent analysis. To avoid the curse of dimensionality, PCA was applied to reduce the feature dimension, with $95 \%$ of variance preserved.

2) Classification based on Subclass Discriminant Analysis: LDA is a commonly-used classification method due to its simplicity and effectiveness. However, since LDA assumes that each class distribution follows a Gaussian distribution, it is not suitable for non-Gaussian problems. Subclass discriminant analysis (SDA) is a variant of LDA that aims to separate classes at a subclass level rather than at a class level, based on the observation that the data distribution in one class may be a mixture of Gaussians. This is achieved by dividing each class into several subclasses to describe the variance of data of each class in a fine way and then classifying them with optimal boundaries [22]. Since SDA can model non-Gaussian classes as mixtures of Gaussian subclasses, it can overcome the performance degradation of LDA for non-Gaussian problems [23]. Also, SDA has the potential to provide fine subclass information to give insight into original classes (see Fig. 4). Previous research on SDA always emphasized class separability over subclass separability, ignoring the potential meaning and importance of subclasses [22]-[27]. In this study, we proposed an extension of SDA, which divided subclasses in terms of their physical meanings and emphasized subclass separability as much as class separability. Concretely, we subdivided the data of each finger gesture into a few sub-gestures representing different wrist rotation positions and classified these sub-gestures to achieve simultaneous prediction of finger gestures and wrist rotations.

2.1) Subclass Divisions: How to divide each class into different subclasses is a crucial problem in SDA. Different unsupervised algorithms have been attempted in this field, including K-means clustering [25], [28], dynamic cluster formation [26], Gaussian mixture model [29], hierarchical clustering [24], nearest neighbor (NN) clustering [22], and valley seeking algorithm [27]. However, these unsupervised methods usually clustered data in terms of their inherent similarity, overlooking the physical meanings of the clustered data. In this study, we proposed an IMU-supervised subclass separation method (IMU-SDA), to subdivide the data of each finger gesture $C_{i}(i=1,2, \ldots, 8)$ into $n$ subclasses $C_{i j}(j=1,2, \ldots, n)$ according to the wrist rotation angles. Furthermore, to get rid of the dependence on IMU sensors, a linear relationship between the first principal component of the ultrasound features (PC1) and the wrist rotation angle was built, accomplishing unsupervised PC1-driven subclass divisions (PC1-SDA). Additionally, the unsupervised NN clustering (NN-SDA) was utilized as a comparison [22].

Considering the importance of wrist pronation, wrist supination, and natural wrist position in practical application, the 

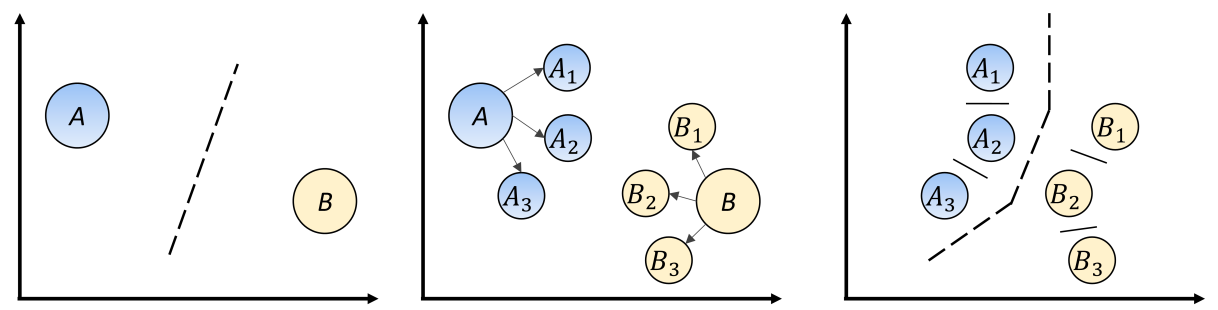

Fig. 4. Illustration of SDA algorithm, where class number and subclass number are 2 and 3 , respectively. The classification of subclasses can give extra information about original classes, and the classification boundaries between original classes (i.e. $A$ and $B$ ) can be non-linear in this way.

number of subclasses was set to 3 in this study. And the subdivision of each class $C_{i j}(j=1,2,3)$ was defined as follows

$$
\begin{aligned}
& C_{i 1}: 0 \leq \alpha<l \\
& C_{i 2}: l \leq \alpha<u \\
& C_{i 3}: u \leq \alpha \leq 1
\end{aligned}
$$

where $\alpha$ represents the normalized wrist rotation angle (derived by IMU or PC1), $l$ and $u$ are parameters for wrist position segmentation, $C_{i 1}, C_{i 2}, C_{i 3}$ represent data from wrist pronation, natural wrist position, and wrist supination, respectively. Empirically, the values of $l$ and $u$ were selected as 0.25 and 0.75 , respectively.

2.2) Subclass Classification: In contrast to previous studies that utilized Fisher's LDA for dimensionality reduction and Knearest neighbors (KNN) classifier for classification [24], [25], we employed a Bayesian linear classifier to classify all the subclasses $C_{i j}(i=1,2, \ldots, 8 ; j=1,2,3)$ and then mapped the subclasses into original classes $C_{i j} \rightarrow C_{i}(i=1,2, \ldots, 8 ; j=$ $1,2,3)$.

Two types of accuracy metrics were defined for assessing the classification performance: finger gesture classification accuracy $\left(A c c_{f}\right)$ and simultaneous wrist rotation classification accuracy $\left(A c c_{w}\right)$.

The finger gesture classification accuracy $\left(A c c_{f}\right)$ was defined as

$$
A c c_{h}=N_{f} / N
$$

where $N$ is the number of test samples and and $N_{f}$ is the correctly recognized finger gestures. The predicted label $C_{i j}$ is correctly recognized into actual label $C_{\bar{i} \bar{j}}$ as long as $i=\bar{i}$ no matter $j=\bar{j}$ or not.

The wrist rotation classification accuracy $\left(A c c_{w}\right)$ was defined as

$$
A c c_{h}=N_{w} / N
$$

where $N$ is the number of test samples, and $N_{w}$ is the number of correctly recognized wrist rotations. The predicted label $C_{i j}$ is correctly recognized into actual label $C_{\bar{i} \bar{j}}$ only if $i=\bar{i}$ and $j=\bar{j}$.

To simulate the real-time application, the first two thirds of data of each gesture were used for model calibration (20 seconds) and the remaining was used for testing (10 seconds) during the offline analysis.
3) Continuous Wrist Rotation Estimation: By SDA, only discrete wrist rotation positions can be predicted instead of continuous wrist rotations. Considering that forearm muscle morphology varies accordingly with wrist rotations, it is likely to extract continuous wrist rotation information from ultrasound signals. According to the offline analysis, the PC1 of selected ultrasound features was approximately linear to the wrist rotation angle, hence the PC1 was used for online proportional wrist rotation control.

The PCA was conducted for the data of all the finger gestures together; therefore, we created a unified PCA projection matrix $(W)$ for the data of all the finger gestures. Then, we calculated the maximum and minimum $\mathrm{PC} 1$ values of each gesture $i$ $\left(P C 1_{\max , i}, P C 1_{\min , i}, i=1,2, \ldots, 8\right)$ and recorded the average maximum and minimum $\mathrm{PC} 1$ values of all the finger gestures $\left(P C 1_{\max }=\frac{1}{8} \sum_{\mathrm{i}=1}^{8} P C 1_{\max , i}, P C 1_{\min }=\frac{1}{8} \sum_{\mathrm{i}=1}^{8} P C 1_{\min , i}\right)$. During the online test, the ultrasound feature was first projected into a low-dimensional space by PCA. Then, the derived PC1 was normalized by the $P C 1_{\min }$ and $P C 1_{\text {max }}$. In this way, the $\mathrm{PC} 1$-based wrist rotation control was independent of the finger gesture control, since both the projection matrix of PCA $(W)$ and the normalization boundaries $\left(P C 1_{\min }, P C 1_{\max }\right)$ of the $\mathrm{PC} 1$ were independent of finger gestures.

For the continuous wrist rotation estimation, the coefficient of determination $\left(R^{2}\right)$ was used to assess the performance.

$$
R^{2}=1-\frac{S S_{\mathrm{res}}}{S S_{\text {tot }}}=1-\frac{\sum_{\mathrm{i}}\left(\hat{y}_{i}-y_{i}\right)^{2}}{\sum_{i}\left(\bar{y}-y_{i}\right)^{2}}
$$

where $S S_{\text {res }}$ is the sum of squares of residual errors, $S S_{t o t}$ is the total sum of squares, $y_{i}$ is the actual label of sample $i, \hat{y}_{i}$ is the predicted label,and $\bar{y}=\frac{1}{n} \sum_{i=1}^{n} y_{i}$. The maximal $R^{2}$ at perfect estimation is one.

\section{E. Statistical Analysis}

One-way repeated-measure analysis of variance (ANOVA) and Mann-Whitney $U$ test were used to compare the performance of different classification models (i.e. LDA and SDA) and subclass division methods (i.e. NN-SDA, IMUSDA, and PC1-SDA). If analyzed data followed a normal distribution, one-way ANOVA would be applied otherwise Mann-Whitney U test would be applied. In addition, two-way repeated-measure ANOVA was used to evaluate the performance of different classification models (i.e. LDA, SDA, and 
quadratic discriminant analysis (QDA)) and sensor positions (i.e. Channel 1 to Channel 8). When ANOVA revealed a significant difference, Tukey method was used for the posthoc comparison. Statistical significance was set to $p=0.05$.

\section{RESULTS}

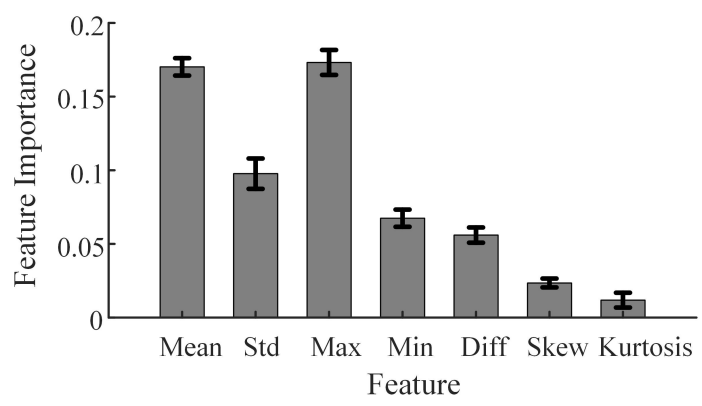

Fig. 5. Importance of selected features, which is derived by the Random Forest algorithm. The data are averaged across different subjects.

\section{A. Feature Selection}

The importance of selected features derived by the Random Forest algorithm is shown in Fig. 5. It was found that the mean $\left(f_{1}\right)$ and max $\left(f_{3}\right)$ were the most important features, followed by the $\operatorname{std}\left(f_{2}\right), \min \left(f_{4}\right), \operatorname{sum}(\operatorname{abs}(\operatorname{diff}(\cdot)))\left(f_{5}\right)$, skewness $\left(f_{6}\right)$, and kurtosis $\left(f_{7}\right)$. Considering the superior performance of the mean $\left(f_{1}\right)$ and std $\left(f_{2}\right)$ features and their complementary properties, the combined mean and std (MSD) features were selected for the following analysis.

\section{B. Effectiveness of SDA}

The performance of IMU-SDA for the simultaneous wrist/hand motion prediction is depicted in Fig. 6, where LDA was used as a benchmark. For the finger gesture recognition, the performance of SDA and LDA was comparable $(p=$ 0.17). However, SDA can achieve accurate wrist rotation classification at the same time. Overall, the average finger gesture classification accuracy and wrist rotation classification accuracy of SDA were $99.89 \% \pm 0.28 \%$ and $95.2 \% \pm 4.9 \%$, respectively.

\section{Principal Component Representation of Wrist Rotation}

Fig. 7 shows the relationship between the wrist rotation angle and the PC1 of MSD features, where PCA was conducted for the data of all the finger gestures together. It was found that the PC1 was approximately linear to the wrist rotation angle regardless of finger gestures. The linear relationship between the $\mathrm{PC} 1$ and wrist rotation angle is summarized in Table I, with an average $R^{2}$ of 0.908 and a normalized root mean squared error $(n R M S E)$ of 0.09 across different subjects.

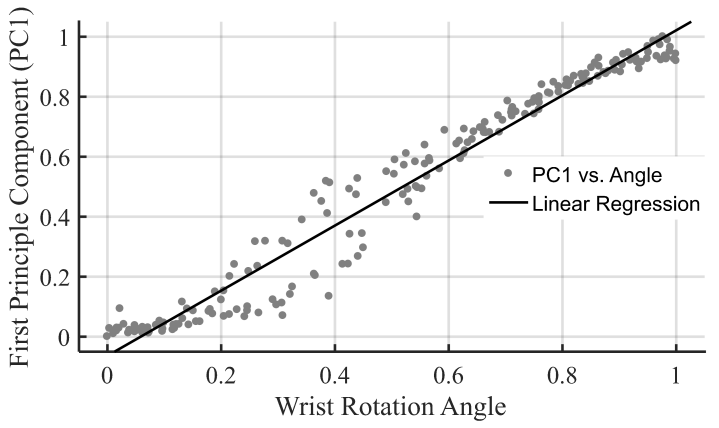

Fig. 7. Relationship between the normalized wrist rotation angle and the normalized PC1 of mean and standard deviation (MSD) features for a representative subject.

TABLE I

LiNEAR REgRESSION BETWEEN THE WRIST Rotation ANGLE AND THE PC1 OF THE MSD FEATURES.

\begin{tabular}{cccccccccc}
\hline \hline & $S 1$ & $S 2$ & $S 3$ & $S 4$ & $S 5$ & $S 6$ & $S 7$ & $S 8$ & $A V E$ \\
\hline$R^{2}$ & 0.906 & 0.885 & 0.905 & 0.888 & 0.939 & 0.945 & 0.921 & 0.877 & 0.908 \\
$n R M S E$ & 0.085 & 0.103 & 0.092 & 0.086 & 0.073 & 0.078 & 0.086 & 0.114 & 0.090 \\
\hline \hline
\end{tabular}

\section{Comparison of Subclass Division Methods}

Now that the PC1 was linear to the wrist rotation angle, the segmentation of wrist rotations using PC1 was incorporated into SDA (PC1-SDA). The comparison of IMU-SDA, PC1SDA, and NN-SDA is shown in Fig. 8. In terms of the finger gesture classification, the performance of IMU-SDA, PC1SDA, and NN-SDA was comparable. For the wrist rotation classification, the IMU-SDA outperformed the NN-SDA significantly $(p<0.001)$, and the PC1-SDA outperformed the IMU-SDA significantly $(p<0.001)$.

\section{E. Online Wrist/Hand Motion Prediction}

The online wrist/hand motion prediction performance is depicted in Fig. 9. When using SDA (IMU-SDA), the average finger gesture classification accuracy was $99.2 \pm 1.2 \%$ under dynamic wrist rotations, and the simultaneous wrist rotation

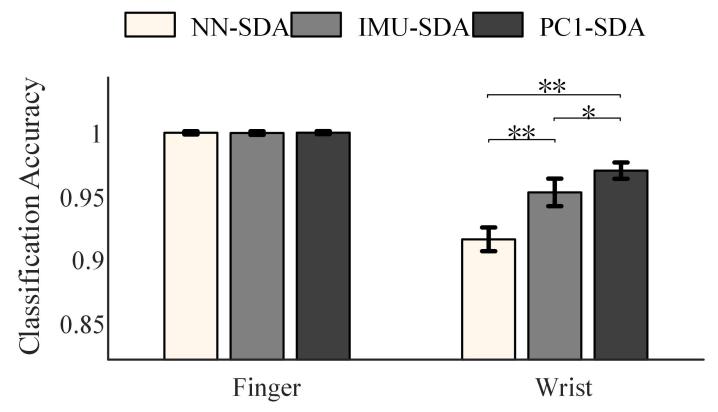

Fig. 8. Comparison of different subclass division methods. NN-SDA, IMUSDA, and PC1-SDA represent nearest neighbor clustering based subclass division, IMU-based subclass division, and PC1-based subclass division, respectively. The NN-SDA and PC1-SDA are unsupervised, but the IMU-SDA requires an external IMU sensor for the supervised subclass division. The data are averaged across different subjects and finger gestures. ${ }^{*} p<0.05$, ** $p$ $<0.001$. 


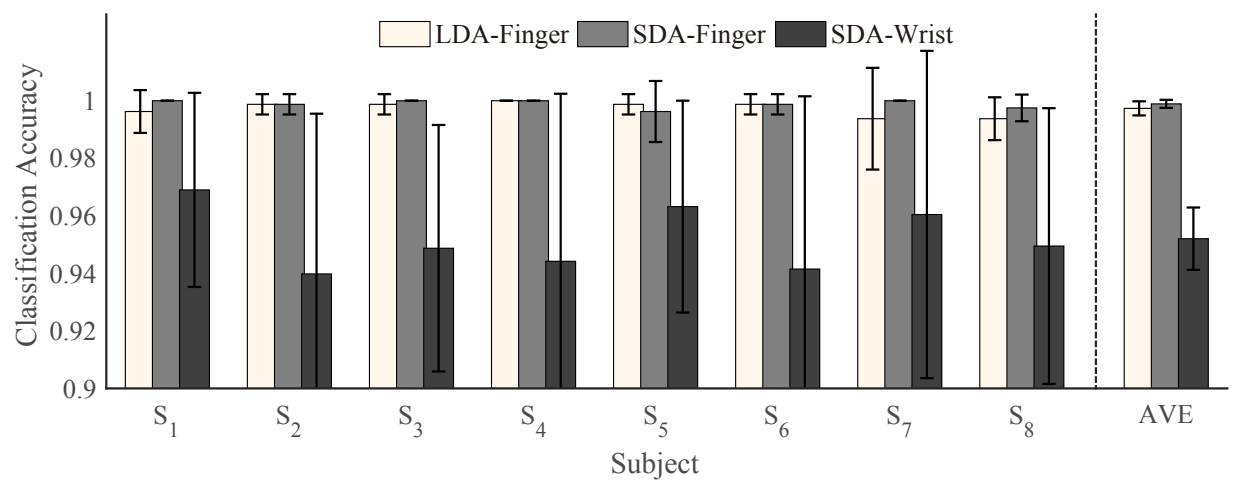

Fig. 6. Simultaneous wrist/hand motion prediction performance of inertial measurement unit (IMU) based SDA, where the linear discrimination analysis (LDA) is used as a benchmark. LDA-Finger and SDA-Finger denote the finger gesture classification accuracy of LDA and SDA, respectively. SDA-Wrist denotes the simultaneous wrist rotation classification accuracy of SDA. The data are averaged across different finger gestures.

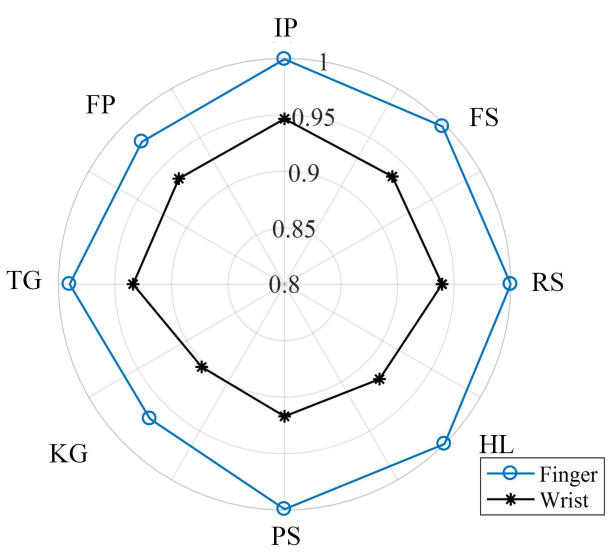

(A) SDA

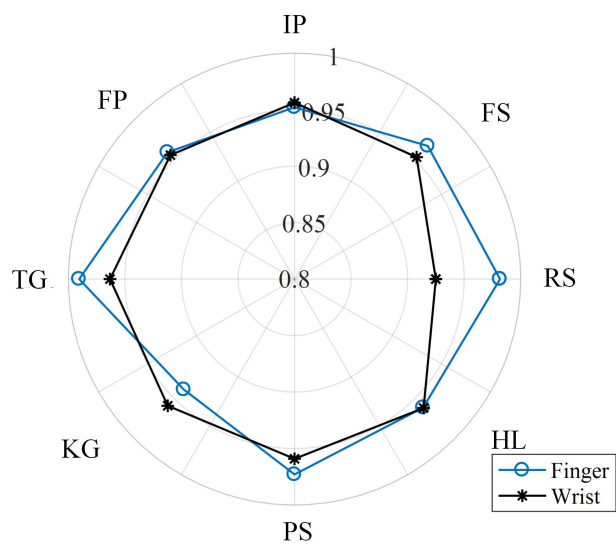

(B) PC1-LDA

Fig. 9. Online simultaneous wrist/hand motion prediction performance of SDA and PC1-LDA. (A) Online finger gesture and wrist rotation classification accuracy of IMU-SDA. (B) Online finger gesture classification accuracy of LDA, and the simultaneous wrist rotation tracking accuracy $\left(R^{2}\right)$ of the PC1 of MSD features. The data are averaged across different subjects and finger gestures.

classification accuracy was $92.8 \pm 1.4 \%$ (see Fig. 9a). When using PC1 for proportional wrist position estimation and LDA for finger gesture classification (PC1-LDA), the average finger gesture classification accuracy was $96.5 \pm 1.7 \%$ under dynamic wrist rotations, and the simultaneous wrist rotation tracking precision $\left(R^{2}\right)$ was $0.954 \pm 0.012$ (see Fig. 9b).

The online wrist rotation tracking performance of a representative subject is shown in Fig. 10. It was clear that the tracking curves were in good agreement with the target waveform, regardless of if we used SDA for discrete wrist rotation estimation or $\mathrm{PC} 1$ for continuous wrist rotation tracking.

\section{DISCUSSION}

Simultaneous prediction of wrist/hand motion provides a natural approach to artificial hand manipulation. Due to the crosstalk in sEMG recordings, it is hard to discriminate activations of wrist/hand motions using sEMG. By contrast, ultrasound sensing has the potential to distinguish finger and wrist motions simultaneously, since finger- and wrist- related muscles are seated in different depths and discernable by ultrasound. This paper provides a scheme to predict wrist/hand motions simultaneously via ultrasound. As wrist rotation and

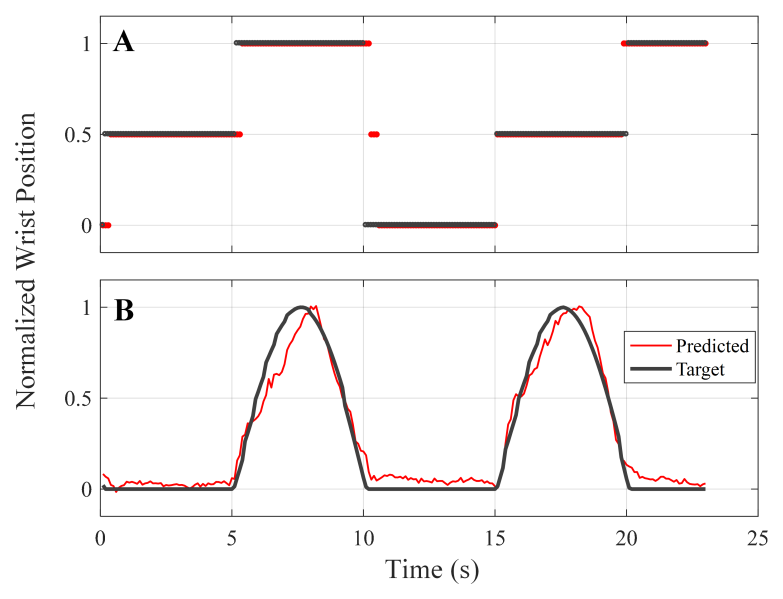

Fig. 10. Online wrist position tracking performance of the RS gesture for a representative subject. (A) Discrete wrist position tracking using SDA. (B) Continuous wrist position tracking using the PC1 of MSD features.

finger grasps are crucial for practical applications, these motions were targeted in this study [30], [31]. In addition, A-mode ultrasound was utilized due to its wearability. We 
proposed a novel SDA algorithm and a PCA strategy for the prediction of simultaneous wrist rotation and finger gestures. Offline evaluation demonstrated that SDA can achieve accurate finger gesture classification under dynamic wrist rotations, accompanied by remarkable wrist rotation classification (see Figs. 6 and 8). Online testing validated that the proposed approach is capable of attaining desirable wrist/hand control in real-time, either in a discrete manner by SDA or in a continuous manner by PC1-LDA (see Fig. 9). This is the first study to develop an algorithm to decode simultaneous wrist/hand motions from ultrasound signals, paving the way for musculature-driven prosthesis manipulation.

\section{A. Parameter Optimization of SDA}

There are two parameters in the proposed SDA algorithm, $l$ and $u$, which are responsible for the subclass division of wrist rotations. The influence of these parameters on the classification performance is depicted in Fig. 11, where $l$ ranged from 0.1 to 0.3 and $u$ ranged from 0.7 to 0.9 . It was found that the best performance can be achieved as $l=0.23$ and $u=0.74$, which approximated our empirical parameters $(l=0.25, u=0.75)$. Note that the optimal normalized angle ranges of wrist pronation $(0 \leq \alpha<0.23)$ and wrist supination $(0.74 \leq \alpha \leq 1)$ were smaller than natural wrist position $(0.23 \leq \alpha<0.74)$. The potential reason for this was that subjects were accustomed to holding the wrist in pronation and supination for a while, but varied the wrist positions rapidly between them during the dynamic training, resulting in the number of sampling data from wrist pronation and wrist supination being larger than that from natural wrist position. Therefore, assigning more samples (larger angle range) to the natural wrist position would improve the classification performance of wrist rotations.

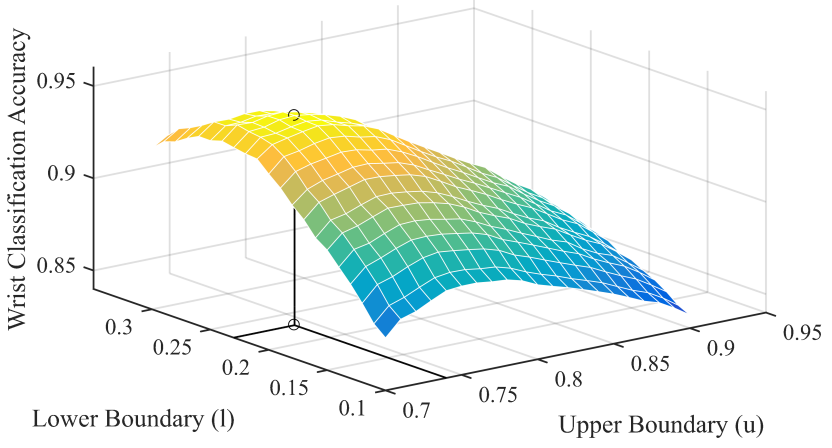

Fig. 11. Relationship between wrist rotation segmentation parameters (i.e. $l$ and $u$ ) in SDA and the wrist rotation classification performance of SDA, where IMU-SDA is utilized as a representative. The data are averaged across different subjects and finger gestures.

\section{B. Comparison of $L D A, S D A$, and $Q D A$}

In order to account for the classification mechanism of SDA, we further compared the finger gesture classification performance of LDA, SDA (IMU-SDA herein), and QDA when using single channel ultrasound (see Fig. 12). Twoway ANOVA shows that there was no significant interaction between the classification methods and sensor positions, and post-hoc analysis showed that the performance of SDA was significantly better than LDA but significantly worse than QDA. The potential reason for this was that SDA can extend the linear classification boundaries to be non-linear hyperpolygons (see Fig. 13) and thus can improve the generalization ability of LDA. Nevertheless, the classification boundaries of QDA are hyper-quadric, with better non-linear characteristics than the hyper-polygonal boundaries in SDA.

While QDA was superior to SDA in finger gesture classification accuracy, it cannot provide the wrist rotation information that SDA does. Moreover, the computation cost of QDA is higher than SDA. The computation costs of LDA, SDA, and QDA for a frame of ultrasound signals were $0.008 \mathrm{~ms}$, $0.017 \mathrm{~ms}$, and $0.020 \mathrm{~ms}$, respectively $(3.2-\mathrm{GHz}$ Intel Core i5-3470 CPU, 8-GB memory, Matlab 2016Rb). That is because QDA determines quadratic classification boundary using class-wise covariance matrices, while LDA/SDA eliminates quadratic terms using a unified covariance matrix for all classes [32]. If training samples are inefficient, the covariances of individual classes might be singular. In this case, QDA will be invalid but LDA/SDA will remain effective. Overall, SDA incorporated nonlinearity into LDA and provided extra subclass information.

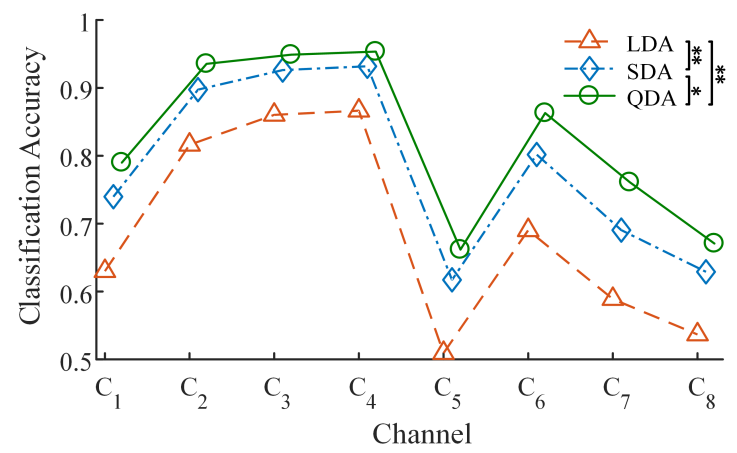

Fig. 12. Comparative analysis of LDA, SDA and quadratic discriminant analysis (QDA) on the finger gesture classification when using single channel ultrasound sensing. The sensor positions are illustrated in Fig. 2. $* p<0.05$, $* * p<0.001$.

\section{Principal Component Representation of Wrist Rotation}

While IMU-SDA can achieve accurate recognition of simultaneous wrist/hand motions, it relies on an IMU sensor for the
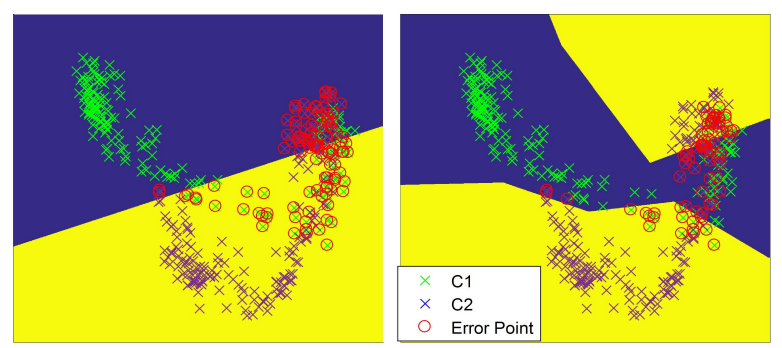

Fig. 13. Comparison of LDA (Left) and SDA (Right)-based classification boundaries. 
subclass divisions, which hinders its clinical applicability. It is vital to create unsupervised subclass division in SDA, by which simultaneous wrist/hand control can be accomplished solely by ultrasound. To maintain the physical meanings of subclasses for representing wrist rotation positions, a feature that represents wrist rotation angle was necessary for the subclass divisions. As shown in Fig. 7, the PC1 of MSD features can represent wrist rotation position, and it was independent of finger gestures. That is because muscle movement is more intense during wrist rotations compared to finger pinches; hence, ultrasound signals varied more significantly during wrist rotations. According to the principle of PCA, the largest signal variance would be reflected in the first dimension; therefore, the PC1 of ultrasound features was related to the wrist rotation. Since the PC1 could be an indicator of wrist rotation angle, we incorporated it into SDA for the subclass divisions. Results showed that the PC1-SDA was comparable to the IMU-SDA on finger gesture classification and superior to IMU-SDA on wrist rotation classification. The potential reason for this is that there is no delay between the PC1 and ultrasound signals, but a delay might exist between the IMU and ultrasound signals.

As mentioned above, the PC1 was proportional to the wrist rotation angle regardless of finger gestures. Therefore, the PC1 can be used for proportional wrist rotation control during multi-DoF finger gesture control. This has been demonstrated in the online wrist/hand control task, with an average wrist rotation tracking precision $\left(R^{2}\right)$ of $0.954 \pm 0.012$ and a simultaneous finger gesture recognition accuracy of $96.5 \pm$ 1.7\% (see Fig. 9b). The PC1-based wrist rotation estimation was an unsupervised algorithm. Compared to regression-based methods, it was more training-efficient and easier to be applied in clinical applications. Furthermore, while it was not validated in this study, SDA can be combined with the PC1-based continuous wrist rotation estimation. In this way, accurate finger gesture recognition can be achieved by SDA, and the wrist rotation angles can be accurately estimated simultaneously, either in a discrete manner by SDA or in a continuous manner by the $\mathrm{PC} 1$ selectively.

A limitation of this study is the absence of amputee subjects. However, there is potential to extend this method to amputee prosthesis control, since the effectiveness of ultrasound-based gesture recognition on amputees has been demonstrated [33], and the continuous wrist rotation training strategy in this study is more suitable for amputees compared to previously reported mirrored bilateral training [12].

\section{CONCLUSION}

In this paper, we presented a novel SDA algorithm and a PCA strategy for the prediction of simultaneous wrist rotation and finger gestures via wearable ultrasound. Results showed that the proposed SDA algorithm can achieve accurate finger gesture classification during wrist rotations, accompanied by precise wrist rotation classification. Moreover, the first principal component of selected ultrasound features was linear to the wrist rotation angle regardless of finger gestures. This provides a desirable method for the unsupervised subclass division in
SDA and a promising approach to proportional wrist rotation control during multifreedom finger gesture control, paving the way for future prosthesis control.

\section{REFERENCES}

[1] J. M. Hahne, F. Biessmann, N. Jiang, H. Rehbaum, D. Farina, F. Meinecke, K.-R. Müller, and L. Parra, "Linear and nonlinear regression techniques for simultaneous and proportional myoelectric control," IEEE Transactions on Neural Systems and Rehabilitation Engineering, vol. 22, no. 2, pp. 269-279, 2014

[2] C. Castellini, G. Passig, and E. Zarka, "Using ultrasound images of the forearm to predict finger positions," IEEE Transactions on Neural Systems and Rehabilitation Engineering, vol. 20, no. 6, pp. 788-797, 2012.

[3] N. Jiang, K. B. Englehart, and P. A. Parker, "Extracting simultaneous and proportional neural control information for multiple-DOF prostheses from the surface electromyographic signal," IEEE Transactions on Biomedical Engineering, vol. 56, no. 4, pp. 1070-1080, 2009.

[4] K. Davidge, Multifunction myoelectric control using a linear electrode array. ProQuest, 2008.

[5] A. J. Young, L. H. Smith, E. J. Rouse, and L. J. Hargrove, "A new hierarchical approach for simultaneous control of multi-joint powered prostheses," in 2012 4th IEEE RAS \& EMBS International Conference on Biomedical Robotics and Biomechatronics (BioRob). IEEE, 2012, pp. 514-520.

[6] J. J. Baker, E. Scheme, K. Englehart, D. T. Hutchinson, and B. Greger, "Continuous detection and decoding of dexterous finger flexions with implantable myoelectric sensors," IEEE Transactions on Neural Systems and Rehabilitation Engineering, vol. 18, no. 4, pp. 424-432, 2010.

[7] A. Boschmann, M. Platzner, M. Robrecht, M. Hahn, and M. Winkler, "Development of a pattern recognition-based myoelectric transhumeral prosthesis with multifunctional simultaneous control using a modeldriven approach for mechatronic systems," in Proceedings of the MyoElectric Controls/Powered Prosthetics Symposium Fredericton, New Brunswick, Canada, 2011.

[8] L. H. Smith and L. J. Hargrove, "Comparison of surface and intramuscular EMG pattern recognition for simultaneous wrist/hand motion classification," in 2013 35th annual international conference of the IEEE engineering in medicine and biology society (EMBC). IEEE, 2013, pp. 4223-4226

[9] A. J. Young, L. H. Smith, E. J. Rouse, and L. J. Hargrove, "Classification of simultaneous movements using surface EMG pattern recognition," IEEE Transactions on Biomedical Engineering, vol. 60, no. 5, pp. 1250$1258,2013$.

[10] M. Nowak and C. Castellini, "The LET procedure for prosthetic myocontrol: towards multi-DOF control using single-DOF activations," PloS one, vol. 11, no. 9, p. e0161678, 2016.

[11] C. W. Antuvan and L. Masia, "An LDA-based approach for real-time simultaneous classification of movements using surface electromyography," IEEE Transactions on Neural Systems and Rehabilitation Engineering, 2019.

[12] S. Muceli and D. Farina, "Simultaneous and proportional estimation of hand kinematics from EMG during mirrored movements at multiple degrees-of-freedom," IEEE transactions on neural systems and rehabilitation engineering, vol. 20, no. 3, pp. 371-378, 2012.

[13] J. M. Hahne, M. A. Schweisfurth, M. Koppe, and D. Farina, "Simultaneous control of multiple functions of bionic hand prostheses: Performance and robustness in end users," Science Robotics, vol. 3, no. 19, p. eaat3630, 2018.

[14] Y. Huang, X. Yang, Y. Li, D. Zhou, K. He, and H. Liu, "Ultrasoundbased sensing models for finger motion classification," IEEE journal of biomedical and health informatics, vol. 22, no. 5, pp. 1395-1405, 2018.

[15] J. McIntosh, A. Marzo, M. Fraser, and C. Phillips, "Echoflex: Hand gesture recognition using ultrasound imaging," in Proceedings of the 2017 CHI Conference on Human Factors in Computing Systems. ACM, 2017, pp. 1923-1934.

[16] N. Akhlaghi, C. A. Baker, M. Lahlou, H. Zafar, K. G. Murthy, H. S. Rangwala, J. Kosecka, W. M. Joiner, J. J. Pancrazio, and S. Sikdar, "Real-time classification of hand motions using ultrasound imaging of forearm muscles," IEEE Transactions on Biomedical Engineering, vol. 63, no. 8, pp. 1687-1698, 2016.

[17] J.-Y. Guo, Y.-P. Zheng, H.-B. Xie, and T. K. Koo, "Towards the application of one-dimensional sonomyography for powered upper-limb prosthetic control using machine learning models," Prosthetics and orthotics international, vol. 37, no. 1, pp. 43-49, 2013. 
[18] X. Yang, X. Sun, D. Zhou, Y. F. Li, and H. Liu, "Towards wearable Amode ultrasound sensing for real-time finger motion recognition," IEEE Transactions on Neural Systems and Rehabilitation Engineering, vol. 26, no. 6, pp. 1199-1208, 2018.

[19] J. He, H. Luo, J. Jia, J. T. Yeow, and N. Jiang, "Wrist and finger gesture recognition with single-element A-mode ultrasound signal: A comparison with single-channel surface electromyogram," IEEE Transactions on Biomedical Engineering, 2018.

[20] X. Yang, Z. Chen, N. Hettiarachchi, J. Yan, and H. Liu, "A wearable ultrasound system for sensing muscular morphological deformations," IEEE Transactions on Systems, Man, and Cybernetics: Systems, 2019.

[21] X. Yang, J. Yan, Z. Chen, H. Ding, and H. Liu, "A proportional pattern recognition control scheme for wearable A-mode ultrasound sensing," IEEE Transactions on Industrial Electronics, 2019.

[22] M. Zhu and A. M. Martinez, "Subclass discriminant analysis," IEEE Transactions on Pattern Analysis and Machine Intelligence, vol. 28, no. 8, pp. 1274-1286, 2006.

[23] N. Gkalelis, V. Mezaris, and I. Kompatsiaris, "Mixture subclass discriminant analysis," IEEE Signal Processing Letters, vol. 18, no. 5, pp. 319-322, 2011.

[24] H. Wan, H. Wang, G. Guo, and X. Wei, "Separability-oriented subclass discriminant analysis," IEEE transactions on pattern analysis and machine intelligence, vol. 40, no. 2, pp. 409-422, 2018.

[25] S. Di Cataldo, A. Bottino, I. U. Islam, T. F. Vieira, and E. Ficarra, "Subclass discriminant analysis of morphological and textural features for hep-2 staining pattern classification," Pattern Recognition, vol. 47, no. 7, pp. 2389-2399, 2014.

[26] F. Oveisi, "Subclass discriminant analysis using dynamic cluster formation for EEG-based brain-computer interface," in 2009 4th International IEEE/EMBS Conference on Neural Engineering. IEEE, 2009, pp. 303306.

[27] M. Zhu and A. M. Martínez, "Optimal subclass discovery for discriminant analysis," in 2004 Conference on Computer Vision and Pattern Recognition Workshop. IEEE, 2004, pp. 97-97.

[28] Y. Fang, D. Zhou, K. Li, Z. Ju, and H. Liu, "Attribute-driven granular model for EMG-based pinch and fingertip force grand recognition," IEEE transactions on cybernetics, 2019.

[29] T. Hastie and R. Tibshirani, "Discriminant analysis by Gaussian mixtures," Journal of the Royal Statistical Society: Series B (Methodological), vol. 58, no. 1, pp. 155-176, 1996.

[30] D. A. Bennett and M. Goldfarb, "IMU-based wrist rotation control of a transradial myoelectric prosthesis," IEEE Transactions on Neural Systems and Rehabilitation Engineering, vol. 26, no. 2, pp. 419-427, 2017.

[31] B. Peerdeman, D. Boere, H. Witteveen, H. Hermens, S. Stramigioli, H. Rietman, P. Veltink, S. Misra et al., "Myoelectric forearm prostheses: state of the art from a user-centered perspective." Journal of Rehabilitation Research \& Development, vol. 48, no. 6, 2011.

[32] M. M.-C. Vidovic, H.-J. Hwang, S. Amsüss, J. M. Hahne, D. Farina, and K.-R. Müller, "Improving the robustness of myoelectric pattern recognition for upper limb prostheses by covariate shift adaptation," IEEE Transactions on Neural Systems and Rehabilitation Engineering, vol. 24, no. 9, pp. 961-970, 2016.

[33] A. S. Dhawan, B. Mukherjee, S. Patwardhan, N. Akhlaghi, G. Levay, R. Holley, W. Joiner, M. Harris-Love, and S. Sikdar, "Proprioceptive sonomyographic control: A novel method of intuitive proportional control of multiple degrees of freedom for upper-extremity amputees," arXiv preprint arXiv:1808.06543, 2018. 